Journal of Computer Science 8 (1): 141-144, 2012

ISSN 1549-3636

(C) 2012 Science Publications

\title{
Analytical Study of Signal-to-Noise Ratio for Visible Light Communication by Using Single Source
}

\author{
Mohammad Syuhaimi Ab-Rahman, Nurain Izzati Shuhaimi, \\ Luqman Al-Hakim Azizan and Mazen Radhe Hassan \\ Department of Electrical, Electronics and System Engineering, \\ Faculty of Engineering and Built Environmental \\ Space Science Institute (ANGKASA), University Kebangsaan, Malaysia
}

\begin{abstract}
Problem statement: In this study, optical wireless communication system using visible light is studied for indoor application. Approach: The process started with the calculation on performance of single source white LED where received power, channel DC gain and quality of data transmission (SNR computation) are identified. Through the simulation results, the SNR performance is directly proportional to angle of incident but inversely proportional with the distance. Results: The result at minimum $\mathrm{SNR}$, which is $15 \mathrm{~dB}$, the maximum distance, is recorded at $3.0785 \mathrm{~m}$ while the other parameter which is angle of incident is recorded at $56.989^{\circ}$. Conclusion: A better performance is needed to improve the reliability of the system because based on the analytical modelling; it proves that this system is expected as indoor communication for next generation.
\end{abstract}

Key words: Optical wireless communication, white LED, single source, Light Emitting Diodes (LEDs), direct detection, Intensity modulation, Bit Error Rate (BER), Signal-toNoise Ratio (SNR)

\section{INTRODUCTION}

Light Emitting Diodes (LEDs) have many advantages compared to other kind of light sources. The main point of LEDs lighting is reduced power consumption, where it emit light much faster after power is applied and it stop emitting light much sooner after power is interrupted, which is especially important in some applications. It also more advantageous compared with conventional lighting methods in terms of high efficiency, less temperature dependent, long life time, robust, small in size, lower voltage, cooler operation and so forth (Rahman et al., 2011). At the same time, Visible Light Communication (VLC), is a new kind of optical wireless communication system which applied high brightness visible LEDs as transmitter to set up an optical free-space link with the receiver for high quality transmission of data. Generally, the VLC system is forming into of high brightness visible LEDs and photodetectors, which established of both transmitter and receiver, respectively (Komine and Nakagawa, 2003).
In this system, Intensity Modulation with Direct Detection (IM/DD) is employed and a directed Lineof-Sight (LOS) link has been considered. Noted that, IM/DD is often applied in applications such as shorthaul optical fiber communications and diffuse indoor wireless optical links where inexpensiveness hardware is consumed (Kahn and Barry, 1997). In this kind of systems, the data is modulated on optical intensity of transmitted light using an optical intensity modulator, where the optical intensity is proportional to the transmitted electrical signal. While in the receiver, direct-detection technique is used in which the photo detector generates an output which is proportional to incident received instantaneous power. VLC can be applied in a various applications for both indoor and outdoor environment. But in this research, the proposed system setup considered an optical wireless communication system utilizing LEDs for indoor environment only, as illustrated in Fig. 1 (Kahn and Barry, 1997).

Corresponding Author: Mohammad Syuhaimi Ab-Rahman, Department of Electrical, Electronics and System Engineering, Faculty of Engineering and Built Environmental Space Science Institute (ANGKASA), University Kebangsaan, Malaysia 


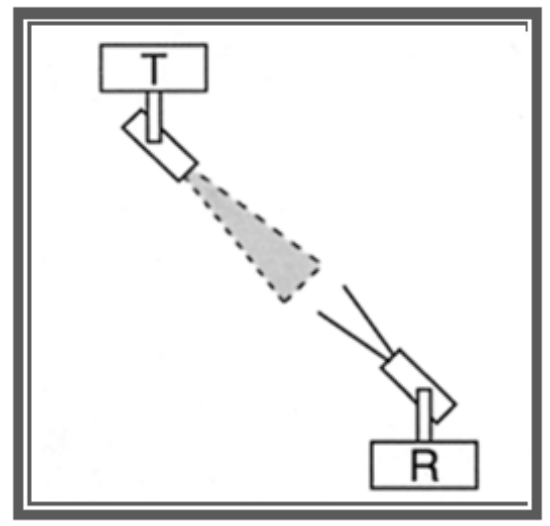

Fig. 1: Directed Link Employs Directional transmitter (LEDs) and receiver (photo detector)

In the other hand, the quality of data transmission in the VLC system can be evaluated through the Bit Error Rate (BER) and Signal-to-Noise Ratio (SNR) computation. The performance of the system is usually rely on external factors such as noise, Intersymbol Interference (ISI), distance between the transmitter and receiver, transmitted power or luminous intensity of LED lights, angle of reflection and so on. This research specifically will look into the above mentioned factors and will concentrate on SNR computation.

\section{MATERIALS AND METHODS}

The process in analyzing the effect of incident and irradiance angle on SNR ratio involves the usage of MathCAD tools. All parameters and calculations that related to this purpose is separated by Part A, B, C and D, where it represents for 'Received Power for Single Source', 'Channel DC Gain', 'Quality of Data Transmission (SNR Computation)' and 'Parameters for Analysis', respectively (Dediang et al., 2007).

Received power for single source: The received power that given by channel $\mathrm{DC}$ gain is:

$$
\operatorname{Pr}:=H(0) . P t
$$

where, $H(0)$ is channel $D C$ gain and $P_{t}$ is the transmitted power. In this analysis, we set the value of transmitted power at $1 \mathrm{~W}$, where the value of it is calculated based on equation below:

$$
\mathrm{Pt}=\mathrm{lim}_{\mathrm{T}=\infty} \frac{1}{2 \mathrm{~T}} \int_{-\mathrm{T}}^{\mathrm{T}} \mathrm{X}(\mathrm{t}) \mathrm{dt}
$$

where, $X(t)$ represents instantaneous input power.

Channel DC gain: Channel DC gain is given by:

$$
\mathrm{H}(\psi, \phi):=\frac{(\mathrm{m}+1) \cdot \mathrm{A} \cdot \cos (\phi)^{\mathrm{p}} \cdot \mathrm{Ts} \cdot \mathrm{g}(\psi) \cdot \cos (\psi)}{2 \cdot \pi \cdot \mathrm{d}^{2}}
$$

where, $\mathrm{m}$ is an order of Lumberton emission, $\mathrm{A}$ is physical area of detector in photo detector, $\Phi$ is the angle of irradiance, Ts is a gain of optical filter, $\mathrm{g}(\psi)$ is the gain of optical concentrator, $\psi$ is the angle of incidence and $\mathrm{d}$ is the distance between transmitter and receiver.

In order to get Lambert an emission, equation below is used:

$$
\mathrm{p}:=\frac{-\ln (2)}{\operatorname{in}(\cos (\phi c))}
$$

where, $\phi c$ is transmitter semiangle which defined at half power.

While the optical concentrator $\mathrm{g}(\psi)$ equation is then given by:

$$
g(\psi):=\mid \begin{aligned}
& \frac{\left(\mathrm{n}^{2}\right)}{(\sin (\psi \mathrm{c}))^{2}} \text { if } 0<\psi<\psi \mathrm{c} \\
& 0 \text { otherwise }
\end{aligned}
$$

where, $\mathrm{n}$ represents the refractive index and we set the value at 1.5.

Quality of data transmission (SNR computation): In the wireless communication, the bandwidth will likely be much higher that data rate, so that the system can operate at very low SNR (Ganapathy and Sagayaraj, 2010).

The SNR is given by:

$$
\mathrm{SNR}=\frac{\gamma^{2} \mathrm{Pr}^{2}}{\sigma_{\text {total }}^{2}}
$$

where, $\gamma$ is the photodiode responsibility (A/W) and $\sigma_{\text {total }}^{2}$ is total noise variance and it is given by this equation:

$$
\sigma_{\text {total }}=\sigma_{\text {thermal }}+\sigma_{\text {shot }}
$$

In order to get total noise variance, the thermal noise variance equation is given by:

$$
\text { other }:=\left(\frac{8 \pi \cdot k \cdot T k \cdot \eta \cdot A \cdot 12 \mathrm{~B}^{2}}{\mathrm{G}}\right)+\left(\frac{16 \pi^{2} \cdot \mathrm{k} \cdot \mathrm{Tk} \cdot \mathrm{T} \cdot \mathrm{A}^{2} \cdot 13 \mathrm{~B}^{2}}{\mathrm{gm}}\right)
$$


Table 1: Parameters for simulation

\begin{tabular}{ll}
\hline Receiver area, A & $5 \times 10^{-4} \mathrm{~m}^{2}$ \\
Filter gain, $\mathrm{T}_{\mathrm{s}}$ & 1 \\
Angle of irradiance, $\phi$ & $15^{\circ}$ \\
Distance, $\mathrm{d}$ & $0.85 \mathrm{~m}$ \\
Angle of incidence, [ & $60^{\circ}$ \\
Transmitter semi angle, $\phi \mathrm{c}$ & $60^{\circ}$ \\
Photodiode responsibility, $\gamma$ & $0.54 \mathrm{~A} / \mathrm{W}$ \\
$\psi \mathrm{c}$ & $60^{\circ}$ \\
Boltzmann's constant, $\mathrm{k}$ & $1.3806488 \times 10^{-23} \mathrm{~J} / \mathrm{K}$ \\
Absolute temperature, $\mathrm{T}_{\mathrm{k}}$ & $300 \mathrm{~K}$ \\
Fixed capacitance of photo & $1.12 \mathrm{u} \mathrm{m}{ }^{-2}$ \\
detector per unit area, $\eta$ & \\
Noise bandwidth factor, $\mathrm{I}_{2}$ & 0.562 \\
$\mathrm{I}_{3}$ & 0.0868 \\
Noise bandwidth, B & $100 \mathrm{MHz}$ \\
Open-loop voltage gain, $\mathrm{G}$ & 10 \\
FET channel noise factor, $\Gamma$ & 1.5 \\
FET transconductance, $\mathrm{g}_{\mathrm{m}}$ & $30 \mathrm{~ms}$ \\
Electronic charge, $\mathrm{q}$ & $1.6 \times 10^{-19} \mathrm{C}$ \\
Background current, $\mathrm{I}_{\mathrm{bg}}$ & $5100 \times 10^{-6} \mathrm{~A}$ \\
$\mathrm{y}$ & $2 \mathrm{~m}$ \\
\hline
\end{tabular}

Where:

$\mathrm{k}=$ Boltzmann's constant

$\mathrm{Tk}=$ Absolute temperature

$\eta=$ Fixed capacitance of photo detector per unit area

$\mathrm{I}_{2}=$ Noise bandwidth factor

$\mathrm{B}=$ Equivalent noise bandwidth

$\mathrm{G}=$ Open-loop voltage gain

$\Gamma=$ FET annel noise factor

$\mathrm{g}_{\mathrm{m}}=$ FET transconductance

In the other hand, shot noise variance is given by:

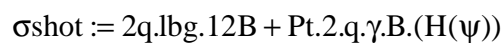

where, $\mathrm{q}$ is the electronic charge and $\mathrm{I}_{\mathrm{bg}}$ is background current.

Parameters for analysis (calculation and simulation): All values that we used in order to get all the calculations above (in part A, B and C) are listed in Table 1 below.

\section{RESULTS}

Figure 2 shows the simulated SNR and angle of incident versus distance characteristic of receiver to determine the signal quality by using single source of light. By looking at Fig. 2, we can see at minimum SNR, which is $15 \mathrm{~dB}$, the maximum distance, is recorded at $3.0785 \mathrm{~m}$. The other parameter, which is angle of incident, is recorded at $56.989^{\circ}$. Theoretically in wireless communication, the acceptable value of SNR is $15 \mathrm{~dB}$ and because of this reason, we set the SNR at this point to analyze the value for angle of incident and distance (Zeng et al., 2008). Noted that, SNR is proportional to angle of incident but it is inversely proportional to the distance.

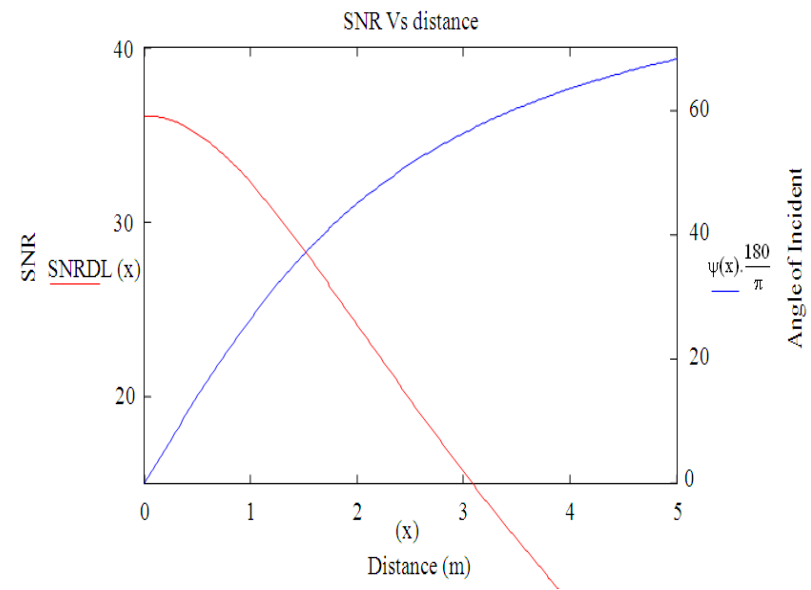

Fig. 2: SNR and angle of incident vs distance

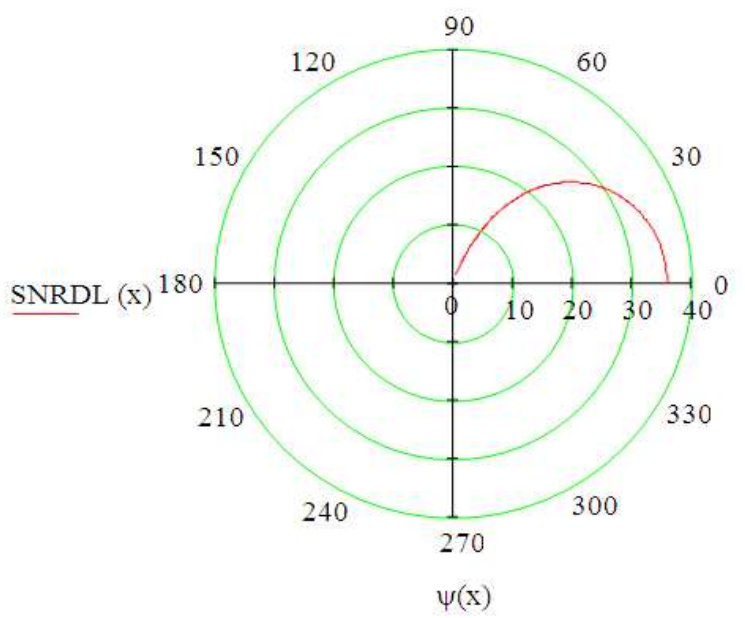

Fig. 3: Polar graph of SNR and angle of incident

A better explanation is shown at Fig. 3 where polar graph is presented. It is also synchronize with result that we get at Fig. 2 which the SNR is $15 \mathrm{~dB}$, thus the angle of incident will be at $56.989^{\circ}$.

\section{DISCUSSION}

For a line of sight configuration, the incident power collected by the photodiode at the receiver end depends on the transmitted power, on the geometry of LED to photodiode, on the channel attenuation and on the receiver model (Komine and Nakagawa, 2004). A simple model for optical communication can be considered as in Fig. 4, where it explained and synchronizes with the result that we get from the graph Fig. 2 on why SNR is directly proportional to angle of incident but inversely proportional with the distance. 


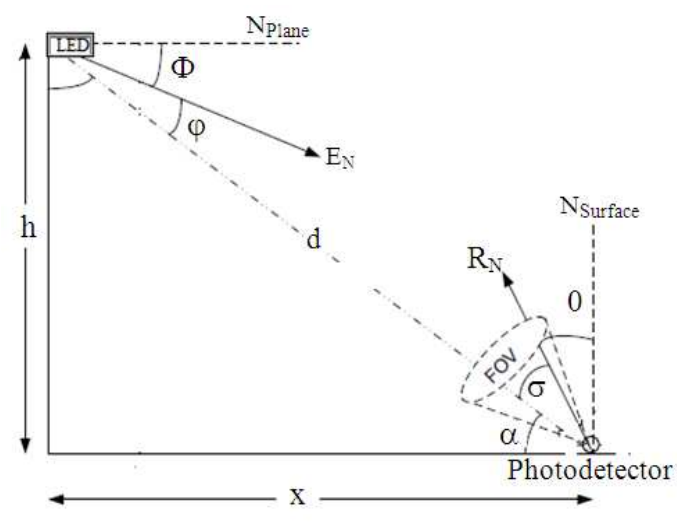

Fig. 4: A simple model for optical communication

From Fig. 4, obviously it has shown a relation between Field Of View (FOV) and received SNR with intersymbol interference. The communication performance is degraded severely by inter symbol interference. In visible light communication system, the LED lights are distributed within a room and the irradiance of light is wide for function of lighting equipment. Therefore, the intersymbol interference is depended on the data rate and FOV of receiver. Noted that when FOV is smaller than $40^{\circ}$, the blind area will exist (Tanaka et al., 2003). The received SNR is required throughout a whole box/room when FOV is $40-60^{\circ}$. Small angle of irradiance gets better performance since the intersymbol interference is decreased.

\section{CONCLUSION}

In this study, in order to observe the incident and irradiance effect on the SNR performance, the twodimensional of transmitter and receiver of VLC application with varying distance has been simulated using the MathCAD tools. Through the simulations, the SNR performance is directly proportional to angle of incident but inversely proportional with the distance. In general, a higher SNR has a better performance due to the larger number of incident angle that available. However, the distance for a better performance must also be decreasing. Thus, to optimise the performance of a system, the angle of incident needs to be customised to suit its application. From the analytical study, a single source of visible light using LED is able to be used for communication and because of this reason, further process and study is continuing for real implementation is achieved.

\section{ACKNOWLEDGEMENT}

The researcher would like to acknowledge University Kebangsaan Malaysia for supporting this research by providing equipment, material and sources of references in accomplishing this research.

\section{REFERENCES}

Dediang, D., K. Xizheng and X. Linpeng, 2007. An optimal lights layout scheme for visible-light communication system. Proceedings of teh 8th International Conference on Electronic Measurement and Instruments, Aug. 16-July 18, IEEE Xploor, Xi'an, pp: 189-194. DOI: 10.1109/ICEMI.2007.4350650

Kahn, J.M. and J.R. Barry, 1997. Wireless infrared communications. Proc. IEEE, 85: 265-298. DOI: $10.1109 / 5.554222$

Komine, T. and M. Nakagawa, 2004. Fundamental analysis for visible-light communication system using LED lights. IEEE Trans. Consumer Elect., 50: 100-107. DOI: 10.1109/TCE.2004.1277847

Komine, T. and M. Nakagawa, 2003. Integrated system of white LED visible-light communication and power-line communication. IEEE Trans. Consumer Elect., 49: 71-79. DOI: 10.1109/TCE.2003.1205458

Komine, T. and M. Nakagawa, 2004. Performance evaluation of visible-light wireless communication system using white LED lightings. Proceedings of the 9th International Symposium on Computers and Communications, June 28-July 1, IEEE Xploor, Japan, DOI: 10.1109/ISCC.2004.1358414

Ganapathy, G. and S. Sagayaraj, 2010. Circumventing picture archiving and communication systems server with hadoop framework in health care services. J. Soc. Sci., 6: 310-314. DOI: 10.3844/jssp.2010.310.314

Rahman, M.S., L.A. Azizan and K. Jumari, 2011. Experimental works on short range communication systems using photovoltaic based receiver. J. Eng. Applied Sci., 5: 268-276. DOI: 10.3923/jeasci.2011.268.276

Tanaka, Y., T. Komine, S. Haruyama and M. Nakagawa, 2003. Indoor visible light data transmission system utilizing white led lights. IEICE Trans. Commun., E86-BP: 2440-2454.

Zeng, L., D. O'Brien and H. Le-Minh, 2008. Improvement of date rate by using equalization in an indoor visible light communication system. Proceedings of the 4th IEEE International Conference on Circuits and Systems for Communications, May 26-28, IEEE Xploor, Shanghai, pp: 678-682. DOI: 10.1109/ICCSC.2008.149 